Acta Cryst. (2002). A58 (Supplement), C126

USE OF BRUCINE TO CRYSTALLISE A DISACCHARIDE FROM A BACTERIAL SOURCE

$\underline{\text { R.O. Gould }}^{1}$ M.D. Walkinshaw ${ }^{1}$ N. Wimmer ${ }^{2}$ P. Kosma ${ }^{2}$

${ }^{1}$ University of Edinburgh ICMB Michael Swann Building Mayfield Road EDINBURGH EH9 3JR UK ${ }^{2}$ Institute of Chemistry, University of Agricultural Sciences, Vienna, A

Because of its very asymmetric structure, the base brucine has long been used to resolve enantiomers of acidic moieties. Here, we have used it to bring about crystallization of an acidic disaccharide otherwise very resistant to crystallization. The disaccharide, containing a carboxylate groups in each octose monomer, was isolated from the lipopolysaccharide of the bacteria Burkholderia cepacia. It shows the substitution of Ko (D-glycero-D-talo-oct-2ulosonic acid) for the normal Kdo (3-deoxy-D-manno-oct-2-ulosonic acid). We have recrystallized it as its dibrucinium salt, a hexahydrate, in which the disaccharide and four of the water molecules are fully ordered. The orthorhombic cell $[a=12.987(2), b=15.362(2), c=33.590(5) \AA]$ shows brucine layers normal to [001], which provide a suitable matrix for the incorporation of the disaccharide. The layer structure of brucine, shown in the diagram, is different from any we have encountered formerly.

Keywords: CARBOHYDRATE, ALKALOID, CRYSTAL PACKING

Acta Cryst. (2002). A58 (Supplement), C126

CONFORMATION ANALYSIS OF MORPHINE ALKALOIDS

H. Petrickova A. Jegorov M. Husak I. Cisarova

Institute of Chemical Technology, Prague Department of Solid State Chemistry Technicka 5 PRAGUE 616628 CZECH REPUBLIC

Morphine alkaloids are used for its analgesic and narcotic effect from of old. These opiates act on central nervous system. They are antagonists or agonists of opioid receptors. Many different derivates of morphine with subtle structural changes may alter the pharmacological activity.

Four new crystal structures, derivates of butorphanol, have been determined by $\mathrm{x}$-ray single crystal diffraction. Molecules of morphine alkaloid usually comprise more or less rigid 4 rings $\mathrm{A}, \mathrm{B}, \mathrm{C}, \mathrm{D}$. They resemble the characteristic T-shape of classical opiates. At present CSD contains 129 structures of morphine alkaloids, but only 27 structures do not possess 4,5epoxy bridge as well as newly reported structures. Conformations of these structures were compared with those four new ones. Ring A is almost planar and rings $\mathrm{B}, \mathrm{C}$ and $\mathrm{D}$ are puckered. Ring $\mathrm{B}$ possesses an envelope conformation, ring $\mathrm{C}$ has a boat conformation distorted towards an envelope and ring $\mathrm{D}$ adopts most often chair conformation. There are also another conformations of ring $\mathrm{D}$ rising commensurate with presence and position of double bond in ring D. The X-ray diffraction analysis gave us evidence that there is no significant difference in the ring conformations among new structures and structures from CSD.

This work was partially supported within the research project CEZ:MSM 223100002 of Ministry of Education of the Czech Republic and projects No. 203/99/0067 and No. 203/99/M037 of the Grant Agency of the Czech Republic.

Keywords: X-RAY STRUCTURE, MORPHINE ALKALOIDS, CONFORMATION
Acta Cryst. (2002). A58 (Supplement), C126

\section{X-RAY STUDY ON POLYMORPHISM OF SOME ERGOPEPTINE} DRUGS

J. Cejka $^{1}$ B. Klepetarova ${ }^{1}$ I. Cisarova ${ }^{2}$ B. Kratochvil ${ }^{1}$ A. Jegorov ${ }^{3}$

${ }^{1}$ Prague Institute of Chemical Technology, Department of Solid State Chemistry Technicka 5 PRAGUE 16628 CZECH REPUBLIC ${ }^{2}$ Charles University, Department of Inorganic Chemistry, Hlavova 8, 12843 Prague 2, Czech Republic ${ }^{3}$ IVAX CR a.s.., Research Unit, Branisovska 31, 37005 Ceske Budejovice, Czech Republic

Ergot alkaloids are well known with their nonselective affinity to various dopamine, serotonine and $\alpha$-adreno receptors. No wonder, they belong to potent pharmaceuticals with a broad range of application in medicine. Utilisation in treatment of elderly patient diseases, stress and anxiety disorders should serve as an example. There is a large variety of structures in Cambridge Structure Database today. However, as can be easily proved, there is lack of systematics and we mostly deal with single structures without any clear feedback to each other.

We have initiated an effort on study of ergot alkaloids polymorphy several years ago. Though there are many gaps left in the system, and, of course, one cannot take in account unpredictable ones, a considerable progress was done. More than one half of published ergot alkaloid structures were solved in our laboratory. The study of ergopeptines, namely ergotamine and ergocristine, their semisynthetic dihydro-derivatives and their mesylate salts crystallized from various liquors will be presented. Comparison of new and known relative structures and general conformational analysis will be performed.

This work was partially supported within the research project CEZ:MSM 223100002 of Ministry of Education of the Czech Republic and projects No. 203/99/0067, No. 203/00/D095 and No. 203/99/M037 of the Grant Agency of the Czech Republic.

Keywords: ERGOT ALKALOIDS POLYMORPHISM ERGOPEPTINE

\section{Acta Cryst. (2002). A58 (Supplement), C126}

POLYMORPHISM IN TRI-P-TOLYL-1,3,5-TRIAZINE

M. Botoshansky A. Lisovskii V. Volkis M.S. Eisen

Technion -Israel Institute of Technology Department of Chemistry Chemistry Department, Qyriat Technion HAIFA 32000 ISRAEL

During the last decade large interest has been devoted to the synthesis of crystalline materials with octupolar non-linear optical behaviors. Although non-centrosymmetric network structures have been published such as tribenzyl isocyanurate (1) and triaryloxytriazine(2) offering low beta-values for the trigonal molecules, tri-p-tolyl- 1,3,5-triazine I, which was expected for it to obtain good hyperpolarizabilities, crystallizes in the centrosymmetric space group Pnma as recently found by Thalladi (3).

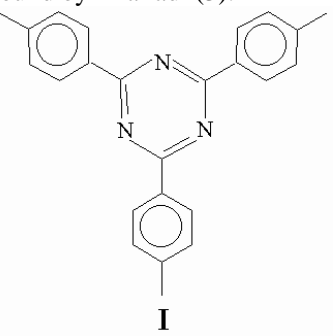

We have recently found a second polymorph of I. The polymorph has also orthorhombic symmetry, but crystallizes in the non-centrosymmetric space group Pmn2 1 [a = 9.458(2) $\AA, \mathrm{b}=21.630(4) \AA, \mathrm{c}=4.573(1) \AA]$. Two of the three crystal cell axes are comparable with first polymorph, but the third axis differs largely. Inspite that the space group Pmn $2_{1}$ is a subgroup of Pnma, these structures cannot be superimposed. In both polymorphs the molecule is bisected by a mirror plane and has similar bonds and angles. The major difference between them is the dihedral angles between the mean plane of the triazine ring and the $\mathrm{a} 0 \mathrm{~b}$ basic plane. In the first polymorph the angle is $58.09^{\circ}$ whereas only $43.37^{\circ}$ is observed for the non-centrosymmetric structure. The absence of the inversion center together with large differences mentioned leads to different packing order and C--H...N interactions. References

(1) Thalladi V.R. et al, Chem. Commun., 1997, 1841-1842

(2) Thalladi V.R. et al, J.Am.Chem.Soc, 1998, 120, 2563-2577

(3) Thalladi V.R. et al, Acta Cryst. 1999, C55, 698-700 